\title{
Color correction matrix for sparse RGB-W image sensor without IR cutoff filter
}

\author{
J.Vaillant ${ }^{* a}$, A.Clouet $^{\mathrm{a}, \mathrm{b}}$, D.Alleysson ${ }^{\mathrm{b}}$ \\ ${ }^{a}$ Univ. Grenoble Alpes, CEA, LETI 38054 Grenoble Cedex 9 France \\ bLaboratoire de Psychologie et NeuroCognition, CNRS UMR 5105, Univ. Grenoble Alpes, \\ 38058 Grenoble, France
}

\begin{abstract}
Unlike photographic image sensors with infrared cutoff filter, low light image sensors gather light over visible and near infrared (VIS-NIR) spectrum to improve sensitivity. However, removing infrared cutoff filter makes the color rendering challenging. In addition, no color chart, with calibrated infrared content, is available to compute color correction matrix (CCM) of such sensors. In this paper we propose a method to build a synthetic color chart (SCC) to overcome this limitation. The choice of chart patches is based on a smart selection of spectra from open access and our own VIS-NIR hyperspectral images databases. For that purpose we introduce a fourth $c_{i r}$ dimension to CIE- $L * a * b *$ space to quantify the infrared content of each spectrum. Then we uniformly sample this $L^{*} a^{*} b^{*} c_{\text {ir }}$ space, leading to 1498 spectra constituting our synthetic color chart. This new chart is used to derive a $3 \times 4$ color correction matrix associated to the commercial RGBWhite sensor (Teledyne-E2V EV76C664) using a classical linear least square minimization.. We show an improvement of signal to noise ratio (SNR) and color accuracy at low light level compared to standard CCM derived using Macbeth color chart.
\end{abstract}

Keywords: Color restitution, low light level, RGB-W, VIS-NIR hyperspectral images, color correction matrix (CCM), synthetic color chart (SCC), CIE $L * a * b *$ space.

\section{INTRODUCTION}

Color correction constitutes an important step in the color image reconstruction pipeline in visible color imaging. Color values of each pixel are spatially interpolated during the demosaicking step [1]. Then the white balance (WB) and color correction matrix (CCM) [2] are applied to each pixel to ensure a good color restitution of the acquired scene (see Figure 1). A large majority of color image sensors contains three color channels Red, Green and Blue (RGB) and an infrared filter in front to only acquire visible range wavelengths [3]. CCM are usually computed over commercially available color charts such as MacBeth ColorChecker (MCC) through a linear mean square error minimization method.

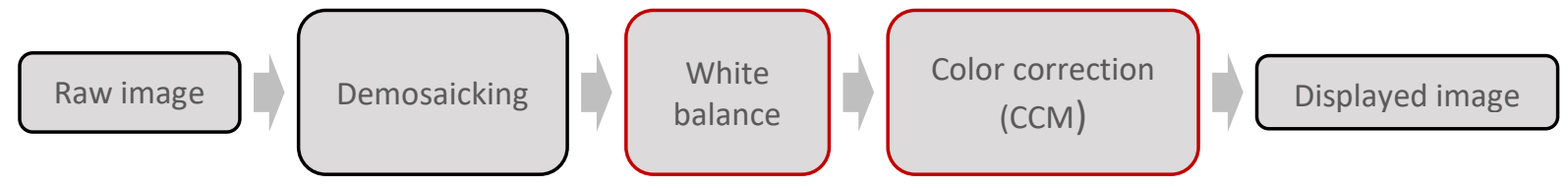

Figure 1. Color restitution flow from raw image to displayed image

Such systems are very efficient in terms of color fidelity in high or mid light level. Nevertheless, using only the visible part of the spectrum limits the signal to noise ratio performance under low light conditions. To adress these applications, monochrome sensors without IR cutoff filters are customary used. So signal to noise ratio (SNR) increased, but at price of color information loss. In case of color image sensor, the absence of IR cutoff filter has detrimental impact on color rendering. Nethertheless we investigate the possibility to capture low light images with decent color rendering using the RGB-White image sensor like the Teledyne-E2V Onyx (EV76C664AMT-RTR) product. This sensor has a monochrome pixel array with sparse RGB pattern, delivering four channels RGBW (see Figure 9).

With such sensors, color correction matrix and white balance are merged into a single $3 \times 4$ matrix converting the raw RGBW output into corrected RGB values to be displayed. In addition, as the sensor is sensitive to near infrared radiation,

*jerome.vaillant@cea.fr 
the standard color charts, like well-known Macbeth chart (ColorChecker® Classic from X-Rite [4]), are not suitable: their properties are defined and controlled only into visible spectrum. This motivates our effort to design a synthetic color chart (SCC) to better constrain the CCM evaluation.

In the following study, we focus on color rendering and we do not address the pixel interpolation part which also requires specific developments. First, we present hyperspectral images databases we consider for the synthetic color chart design. In a second part, we detail the methodology used to choose representative spectra for SCC construction, by introducing a fourth dimension to CIE- $L^{*} a * b *$ space. Then CCM is computed using our chart and spectral sensitivity of Teledyne E2V Onyx sensor. Finally, we compare the impact of standard CCM versus custom CCM on color accuracy and SNR.

\section{REFLECTANCE DATABASE CONSTITUTION}

\subsection{Standard color charts and limitation for VIS-NIR color restitution}

For classical color imaging simulation, reflectance data represented on MCC chart may be used to compute a CCM or adjust white balance (WB) of the imaging device [5]. This chart consists of 24 color patches that represent some physical common colors such as skin or foliage colors... sRGB and $L^{*} a^{*} b^{*}$ values of each patch are given by the manufacturer under respectively D65 and D50 CIE illuminants.

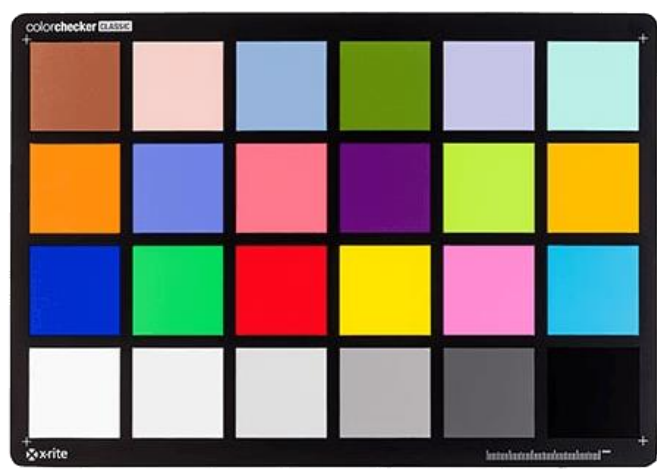

Figure 2. MacBeth ColorChecker chart, from X-Rite [4]

For low light level applications, the infrared cutoff filter is removed from the camera to benefit from the silicon absorbance in this region. In that case, color signals are biased by NIR absorption. To compensate this bias, the NIR content of chart patches need to be known and calibrated. Unfortunately, MCC is not designed to deal with NIR and it is not adapted as reflectance reference for VIS-NIR color restitution. In order to compute a non-biased CCM, we defined a new set of colors with well-known reflectance spectra, based on hyperspectral images databases [6].

\subsection{VIS-NIR reflectance spectra collection}

The new reflectance data are collected from open access hyperspectral images. We took in consideration the Stanford Center for Image Systems Engineering database [7] mostly consisting in skins and landscapes (sky, forest..) reflectance data, the 2009 Multispectral Scene Data from ImageVal [8] consulting, and a set of diverse material reflectance spectra from US Geological Survey [9] (see Figure 3). The hyperspectral images are spatially regularly subsampled to extract at first about 200,000 VIS-NIR reflectance spectra. 

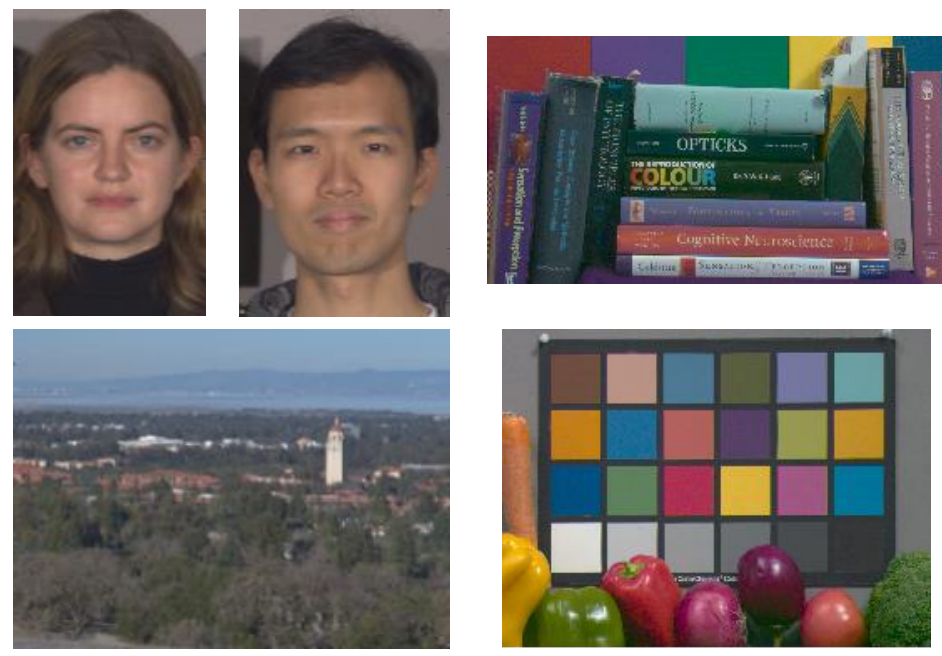

a)

b)

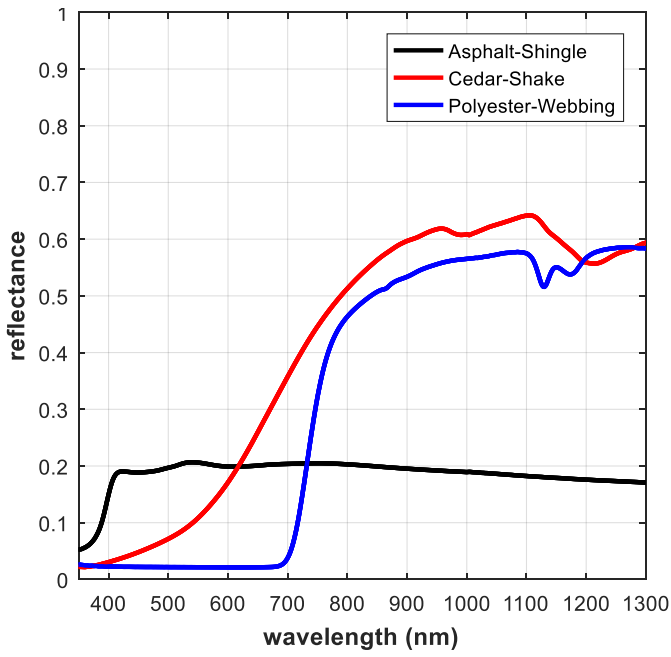

c)

Figure 3. Examples of hyperspectral images and reflectance spectra collected from open access sources: a) SCIEN database b) ImageVal consulting, c) US Geological Survey

To complete the existing databases, we developed our own VIS-NIR hyperspectral image acquisition bench (see Figure 4). The acquisitions are taken under halogen spots with a VIS-NIR monochromatic 10 bits camera and a set of narrow band-pass optical filters allowing to constitute a scene reflectance data cube of 38 spectral narrow bands from $400 \mathrm{~nm}$ to $1050 \mathrm{~nm}$. The image noise is reduced by averaging several frames. For each monochromatic image, dark current and flat field correction are applied. Knowing all physical acquisition parameters such as optical setup, spectral sensitivity of the acquisition sensor and the illuminance spectrum, the reflectance spectra are extracted [5].

a)

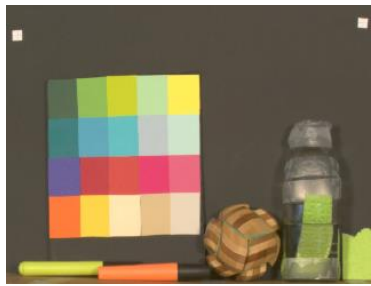

b)

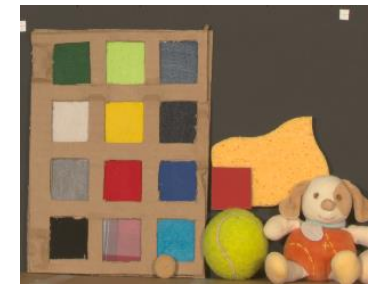

c)

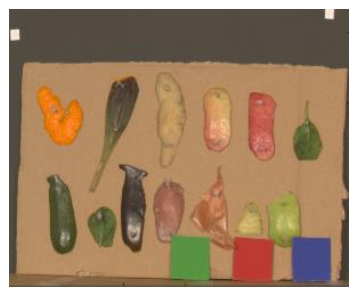

Figure 4. Examples of sRGB reconstitution of hyperspectral images acquired with our own acquisition bench. a) papers, b) textiles, c) vegetables skins

\section{SAMPLING THE DATABASE, TOWARDS A NEW "COLOR" CHART}

After merging reflectance spectra databases, we want to reduce the number of spectra to constitute a suitable synthetic color chart. The selected 200,000 spectra have to be resampled to uniformly cover the chosen "color" space. Firstly we remind $X Y Z$ and $L * a * b *$ spaces formalism, then we detail the extensions we proposed.

\subsection{CIE formalism and MacBeth ColorChecker}

The International Commission on illumination (CIE) developed the $L * a * b *$ color space to approximate the human vision system [10][11]. To calculate $L^{*} a^{*} b^{*}$ coordinates associated to a surface from a reflectance spectrum under known illumination conditions, several mathematical transformations must be done. First of all, reflectance spectra $R(\lambda)$ are projected in CIE $X Y Z$ space under standard CIE illuminant (either D50 or D65) $I(\lambda)$ through the CIE standard observer functions $\bar{x}, \bar{y}$ and $\bar{z}$ (see Figure 6).

$$
\begin{gathered}
X=\frac{1}{N} \int \bar{x}(\lambda) \cdot R(\lambda) \cdot I(\lambda) \cdot d \lambda \quad Y=\frac{1}{N} \int \bar{y}(\lambda) \cdot R(\lambda) \cdot I(\lambda) \cdot d \lambda \quad Z=\frac{1}{N} \int \bar{z}(\lambda) \cdot R(\lambda) \cdot I(\lambda) \cdot d \lambda \\
N=\int \bar{y}(\lambda) \cdot I(\lambda) \cdot d \lambda
\end{gathered}
$$


Next, a non-linear transformation is applied to the $X Y Z$ coordinates to compute $L * a * b *$ coordinates [12]:

$$
L=116 . f_{y}-16 \quad a^{*}=500 .\left(f_{x}-f_{y}\right) \quad b^{*}=200 .\left(f_{z}-f_{y}\right)
$$

For $U=\{X, Y, Z\} U_{W}$ is the white reference coordinate of illuminant

$$
\text { and } f_{U}=\left\{\begin{array}{l}
\left(\frac{U}{U_{w}}\right)^{\frac{1}{3}} \text { if } \frac{U}{U_{w}}>\varepsilon \\
\frac{\kappa \cdot\left(\frac{U}{U_{w}}\right)+16}{116} \text { otherwise }
\end{array} \text { ( } \kappa \text { and } \varepsilon\right. \text { defined by the CIE) }
$$

$L * a * b *$ coordinates associated to the 24 patches of the MCC are provided by the manufacturer under CIE D50 illuminant. Figure 5 presents the $a^{*} b^{*}$ projection of MCC patches. The points are distributed into a range of $[-40,+60]$ on the $a^{*}$ axis and $[-50,+80]$ on the $b^{*}$ axis.
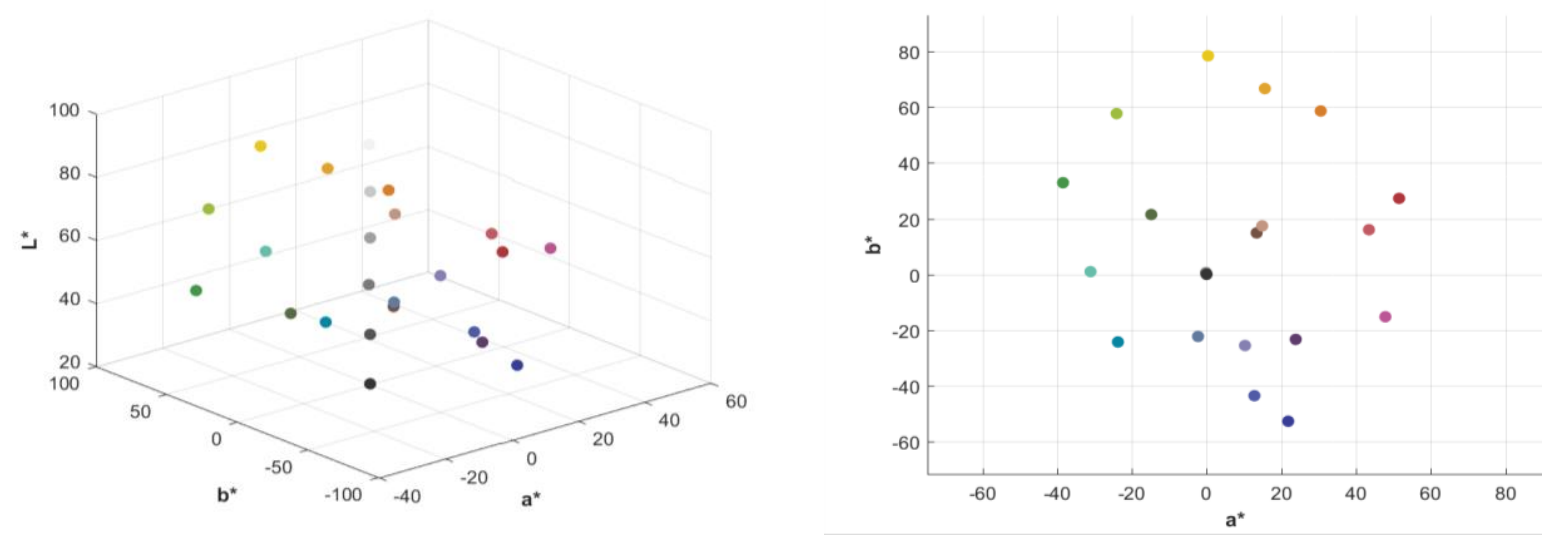

Figure 5. Distribution of the MacBeth ColorChecker chart patches in the $L^{*} a^{*} b^{*}$ space (left) and $a^{*} b^{*}$ plane (right).

\subsection{Near infrared representation, a new 4D $L * a * b * c_{i r}$ space}

Although NIR part is not sensed by human eye and so not represented in $L^{*} a^{*} b^{*}$, we need to project it in a uniform space. In this context, we decided to treat NIR part with the same kind of transformations than visible part adding a new component to the classical CIE $L^{*} a * b *$ space, we called $c_{i r}$.

For that purpose, we add a new component Ir to CIE XYZ space which projects the NIR reflectance data under the chosen illuminant through an $\overline{\imath r}$ function. As mentioned before, $X Y Z$ are usually computed using CIE illuminants which are not defined outside the visible spectrum. In order to use illuminant with NIR content, we replace the CIE D illuminant by its black body equivalent, and $\overline{i r}(\lambda)$ is defined by shifting $\bar{y}(\lambda)$ by $400 \mathrm{~nm}$ toward the NIR domain (Eq. 3). The projection through CIE $\bar{y}$ function gives the visible luminance $Y$ of the considered spectrum. As well as for $Y, I r$ can be considered as a luminance but in NIR domain. This $\overline{\imath r}$ function is represented Figure 6.

$$
\operatorname{Ir}=\frac{1}{N} \int \overline{\operatorname{lr}}(\lambda) \cdot R(\lambda) \cdot I(\lambda) \cdot d \lambda \quad \overline{l r}=\bar{y}\left(\lambda-\lambda_{s}\right) \quad \lambda_{s}=400 \mathrm{~nm}
$$

As for the $X Y Z$ to $L^{*} a^{*} b^{*}$ transformation, a new $c_{i r}$ component is calculated using $Y$ and $\operatorname{Ir}(\operatorname{Eq} 4)$. The $f$ function has been defined $(\mathrm{Eq} 2)$.

$$
c_{i r}=100 \cdot\left(f_{y}-f_{I}\right)
$$

The normalization factor was chosen empirically using the reflectance data collected Section 2 . These spectra are projected in the $L^{*} a * b^{*} c_{i r}$ space under a black body illuminant with a color temperature of $5000 \mathrm{~K}$. The factor is adapted to spread the points over a range about $[-40,+70]$ on $c_{i r}$ alike $a^{*}$ and $b^{*}$ ranges. 


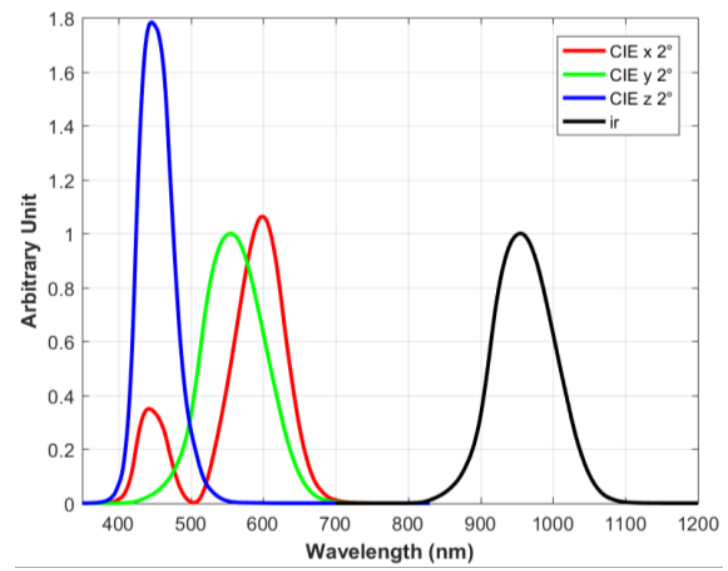

Figure 6. CIE standard observer $\bar{x}, \bar{y}, \bar{z}$ functions for $2^{\circ}$ degree and new $\bar{l}$ function.

\subsection{Resampling of the reflectance database and final synthetic chart}
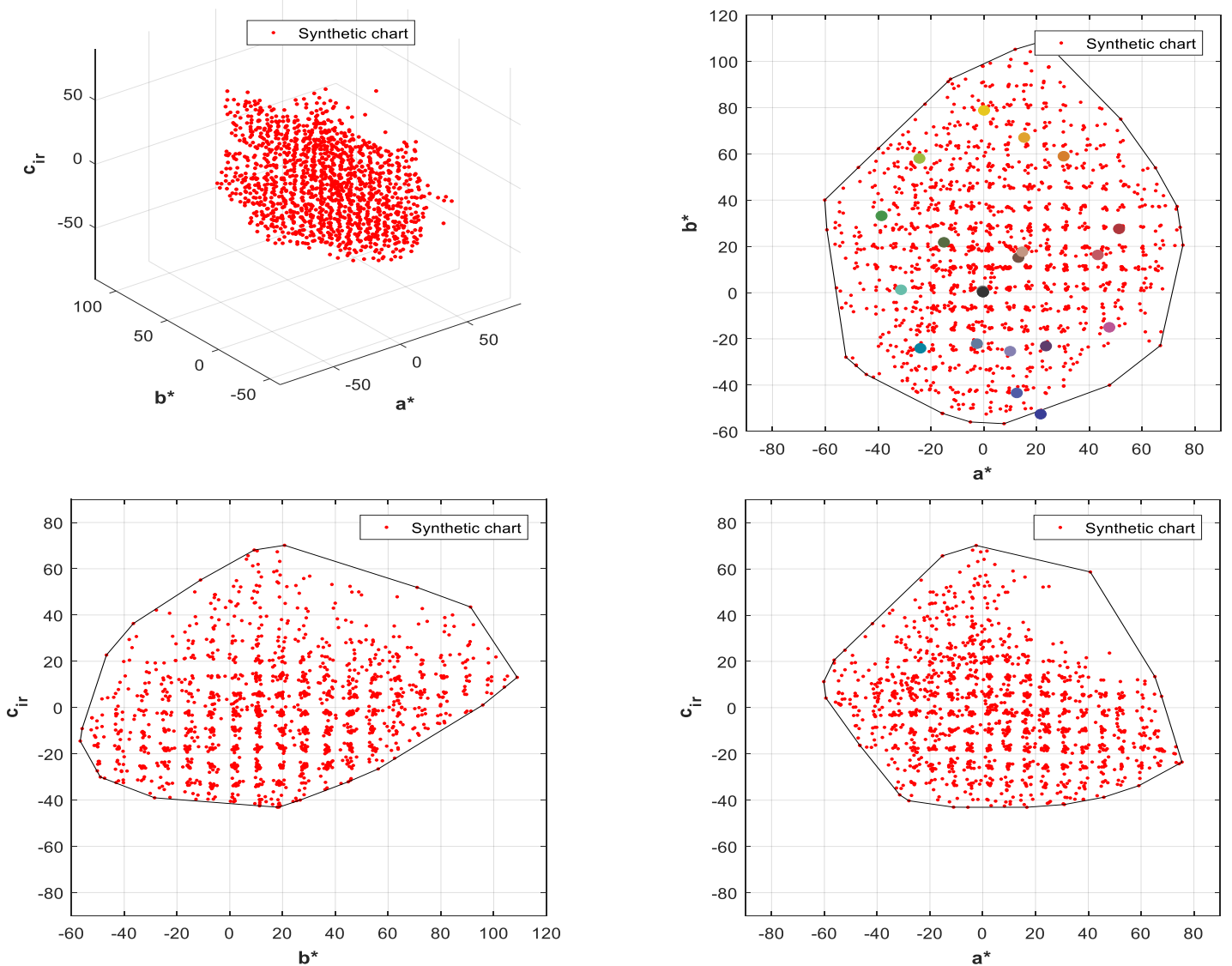

Figure 7. Distribution in CIE like $a^{*} b^{*} c_{i r}$ space (top left) and according to the 3 main planes of the new color 1498 elements color checker under black body (5000K) illuminant. MCC points colorized on top right plot.

A limited number of representative spectra are selected among the 200,000 collected elements to constitute the synthetic color chart. First, we project all spectra into the newly defined $L * a * b * c_{i r}$ space, and we take into account only $a^{*} b * c_{i r}$ components to subsample data: $a^{*}$ and $b^{*}$ are linked to the hue and saturation, and $c_{i r}$ is linked to NIR signal. Then, the $a^{*} b^{*} c_{i r}$ space is uniformly binned into $20 \times 20 \times 20$ elements according to each axe. In every voxels, only the closest point 
to the voxel center is stored. This selection permits to reduce the number of spectra from 200,000 to 1498 which are the components of the new synthetic color chart.

Erreur ! Source du renvoi introuvable. shows the distribution of the $a^{*} b^{*} c_{i r}$ coordinates of the synthetic color chart and the MCC. In $a^{*} b^{*}$ plane, points are uniformly distributed and cover a larger range than the MCC. Figure 8 gives a visual sRGB representation of the synthetic color chart.

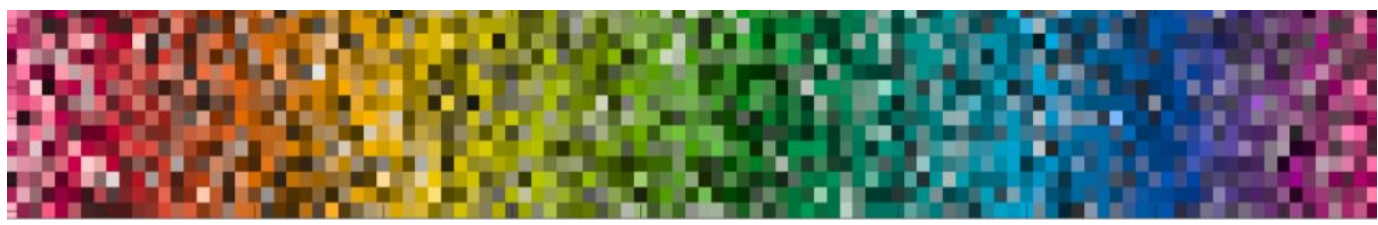

Figure 8. sRGB representation of the synthetic color chart, hue sorted $(a * b *$ plane)

\section{COLOR CORRECTION MATRIX FOR RGB-W LOW LIGHT LEVEL SENSOR}

In this section the performance of the new synthetic color chart has to be tested and compared to the usual color correction method. For that purpose, the physical configuration of the studied sensor must be known to compute a new suitable CCM. We present the sensor we considered, then the CCM computation and finally the results in terms of SNR and color accuracy.

\subsection{Low light level sensor}

The study is focused on the commercial low light sensor Teledyne-E2V Onyx EV76C664AMT. Four channels are used on this sensor: classical RGB and an additional "White" W channel which does not have any color filter.

\section{EV76C664 - QUANTUM EFFICIENCY}

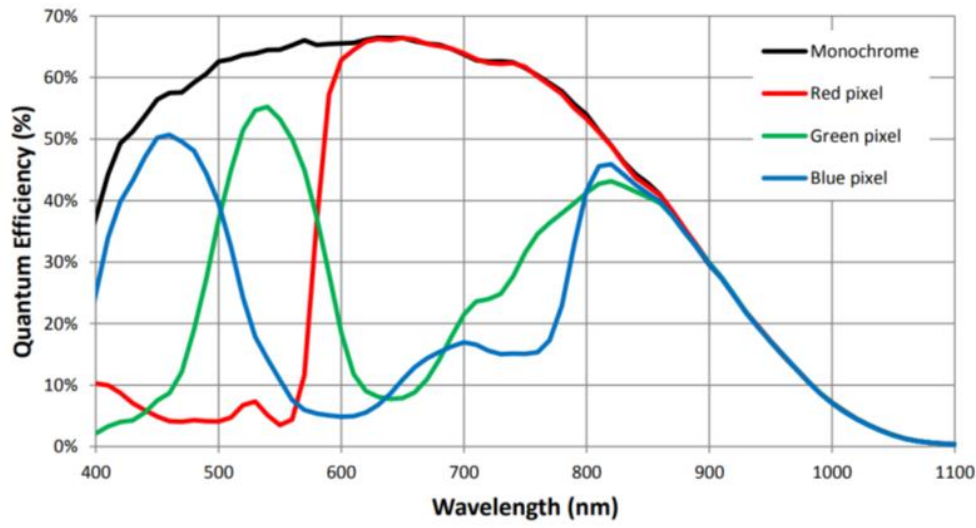

a)

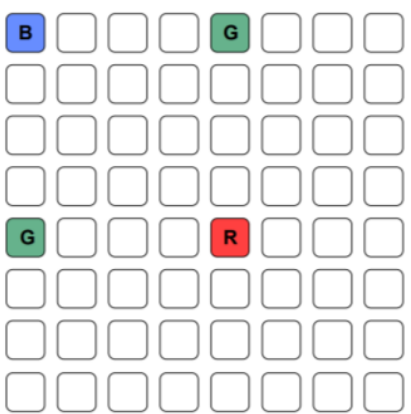

b)

Figure 9. a) Teledyne E2V Onyx E2V76C664 quantum efficiency, b) color filters arrangement (from datasheet). The monochrome channel is called white channel in this paper.

The main issue for low light level acquisition is the poor signal to noise ratio. So maximizing the collected signal is mandatory. Integration time has to be kept low to maintain high framerate in video acquisition. For such application, it is usually done by using the sensor without any infrared filtering to gather all the light on pixels. Obviously, this is detrimental for color fidelity: regarding quantum efficiencies of the channels (Figure 9), it is clear that without infrared filter, the color signals are overestimated due to the NIR transmission of the color filters.

\subsection{Color correction matrix computation, database using}

The camera device signal has its own algebraic space where each element (i.e. color) can be decomposed in a 4 components vector $S=\left[\begin{array}{llll}S_{R} & S_{G} & S_{B} & S_{W}\end{array}\right]^{T}$ (S for Sensor, "T" exponent for transpose). The acquired video sequence is then displayed on a screen which also has an algebraic space. Display spaces are normalized and we choose the sRGB space in this work. The CCM is the linear transformation from the camera space to the displayable normalized space while ensuring the color 
fidelity of the scene. The coefficients of the CCM are computed by linear minimum least square method comparing the results of camera output signals to the expected ones (sRGB) under a chosen illuminant.

For the target, we use our synthetic color chart to compute sRGB target coordinates of each patch: first from spectrum to XYZ under D50 illuminant using Eq. 2, the result is then converted into sRGB coordinates under D65 illuminant (Eq.5) applying Bradford adaptation ( $\left.\mathrm{A}_{\mathrm{Bradford}}\right)[10]$ and the XYZ to RGB matrix $(\mathrm{M})$ and performing gamma correction $(\gamma=$ 2.2).

$$
\left[\begin{array}{l}
R \\
G \\
B
\end{array}\right]=\left(M \cdot A_{\text {Bradford }} \cdot\left[\begin{array}{l}
X \\
Y \\
Z
\end{array}\right]\right)^{1 / \gamma}
$$

Because sRGB is non-linear due to the application of gamma correction $(\gamma=2.2)$, we need to re-linearize targeted RGB coordinates to use the linear minimization formalism. It can be done directly computing RGB coordinates without gamma correction in Eq.5.

For the sensor output, raw RGBW signals are computed considering QE curves shown on Figure 9 over synthetic patches, data are normalized to 255 . Then, these vectors are stored in two matrices, defined in Eq. 6: a 4x1498 matrix called $T$ for targets and a $5 \times 1498$ matrix called $S$ for raw Sensor data. The last row composed of ones is added to compute an intrinsic offset signal to subtract.

$$
T=\left[\begin{array}{c}
T_{R 1} \ldots T_{R 1498} \\
T_{G 1} \ldots T_{G 1498} \\
T_{B 1} \ldots T_{B 1498} \\
1 \ldots 1
\end{array}\right] \quad S=\left[\begin{array}{c}
S_{R 1} \ldots S_{R 1498} \\
S_{G 1} \ldots S_{G 1498} \\
S_{B 1} \ldots S_{B 1498} \\
S_{W 1} \ldots S_{W 1498} \\
1 \ldots 1
\end{array}\right]
$$

The raw correction matrix, denoted $\widehat{M}$, is computed using the linear mean square error minimization, which has a unique analytical solution shown in Eq. 7.

$$
\widehat{M}=\operatorname{argmin}_{M}\left(\mathcal{L}_{2}(T-M . S)\right) \quad \Leftrightarrow \quad \widehat{M}^{T}=\left(S . S^{T}\right)^{-1} \cdot S T^{T}
$$

The $3 \times 4$ CCM and the offset vector are extracted from $\widehat{M}$ as shown in Eq. 8 .

$$
\begin{gathered}
\widehat{M}=\left[\begin{array}{ll}
{[C C M]} & {[V]} \\
{\left[\begin{array}{lll}
0 & \cdots & 0
\end{array}\right]} & 1
\end{array}\right] \\
V_{\text {offset }}=-C C M^{T} .\left(C C M \cdot C C M^{T}\right)^{-1} \cdot V
\end{gathered}
$$

Finally, Eq. 9 explains how to apply the color correction matrix and the offset vector on any raw sensor data $(S=$ $\left.\left[S_{R} S_{G} S_{B} S_{W}\right]^{T}\right)$ to get corrected RGB coordinates $\left(C=\left[C_{R} C_{G} C_{B}\right]^{T}\right)$. Note that gamma correction has to be applied on these corrected values to satisfy sRGB specification and properly display them.

$$
\left[\begin{array}{l}
C_{R} \\
C_{G} \\
C_{B}
\end{array}\right]=\left(C C M \cdot\left[\begin{array}{cc}
S_{R} & V_{\text {offset }}^{R} \\
S_{G} & V_{\text {offset }}^{G} \\
S_{B} & V_{\text {offset }}^{B} \\
S_{W} & V_{\text {offset }}^{W}
\end{array}\right]\right)^{1 / \gamma}
$$

\subsection{SNR and color accuracy results}

The first step of the performance evaluation is to verify SNR improvement for the sensor coupled with our new color correction flow. Noise is usually separated in a chrominance and a luminance components, we consider only the latter. We start computing signal values of the different RGBW channels of the Onyx sensor without infrared filter under black body $(6500 \mathrm{~K})$ illuminant, $20 \mathrm{~ms}$ integration time, f/1 optics, for a grey surface (reflectance of $18 \%$ ). The $3 \times 4 \mathrm{CCM}$ and the offset vectors are computed over the synthetic color chart for the same illuminant, then it is applied to the RGBW values to compute the corrected RGB values. Finally, the luminance signal $Y$ is computed using Eq. 10. Noise variances are calculated, considering photon shot noise of the signal and readout noise of the sensor in order to evaluate the noise variance on the $Y$ channel and compute $\mathrm{SNR}_{Y}$ : the signal to noise ratio on this channel [13][14]. 


$$
Y=0.299 R+0.587 G+0.114 B
$$

For comparison, we consider a sensor dedicated to color photography under high to mid light level: we use RGB QE curves of Onyx product with an IR cutoff filter $\left(\lambda_{\text {cutoff }}=650 \mathrm{~nm}\right)$ placed in front of it. The raw RGB signal values are computed in the same way, under the same conditions. We apply a 3x3 CCM and white balance classically computed over the MacBeth ColorChecker. SNR is also computed on the $Y$ channel. In each case, the SNR computation is done for a wide range of illumination values: from 1 mlux to 100lux. Table 1 shows a comparison between the two configurations in term SNR performance.

Table 1: SNRY performances of sensor with classical or low light configuration

\begin{tabular}{|c|c|c|}
\cline { 2 - 3 } \multicolumn{1}{c|}{} & $\begin{array}{c}\text { Usual color photography } \\
\text { configuration }\end{array}$ & $\begin{array}{c}\text { Low light } \\
\text { configuration }\end{array}$ \\
\hline $\begin{array}{c}\text { Illumination value for } \\
S N R_{Y}=1\end{array}$ & 0.050 lux & 0.015 lux \\
\hline
\end{tabular}

RGBW sensor implementing our color correction is able to deliver $\mathrm{SNR}_{\mathrm{Y}}=1$ for 3 times lower illumination than standard camera. Next, color fidelity should be evaluated too. Obviously, the color photography configuration will deliver far better color $^{\dagger}$ and could be considered as upper bound for color fidelity. So we investigated the impact of color chart used to estimate the CCM. This is done quantitatively by computing the color errors over Synthetic Color Chart patches using CIE $\Delta \mathrm{E}_{2000}$ metric [10]. Table 2 summarizes the results in terms of maximum, mean and minimum errors over the 1498 patches of the synthetic chart. Computing color mismatch over the full 200,000 spectral database confirms that performances are globally increased for a bunch of random spectra, showing that our method is more robust for color rendering when VIS and NIR are acquired with this color camera.

Table 2: Color mismatch (CIE $\Delta \mathrm{E}_{2000}$ error between expected colors and restituted colors over the 1498 patches of the synthetic color chart

\begin{tabular}{|c|c|c|c|c|c|c|}
\hline \multirow[b]{2}{*}{$\begin{array}{l}\text { Applied color correction } \\
\text { matrix }\end{array}$} & \multicolumn{2}{|c|}{$\max \left(\Delta \mathrm{E}_{2000}\right)$} & \multicolumn{2}{|c|}{$\operatorname{mean}\left(\Delta \mathrm{E}_{2000}\right)$} & \multicolumn{2}{|c|}{$\min \left(\Delta \mathrm{E}_{2000}\right)$} \\
\hline & SCC & $\begin{array}{c}\text { Full spectral } \\
\text { database }\end{array}$ & SCC & $\begin{array}{l}\text { Full spectral } \\
\text { database }\end{array}$ & SCC & $\begin{array}{l}\text { Full spectral } \\
\text { database }\end{array}$ \\
\hline $\begin{array}{l}\text { 3x4 CCM computed } \\
\text { using MacBeth } \\
\text { ColorChecker chart }\end{array}$ & 33.18 & 49.24 & 10.15 & 22.69 & 2.24 & 1.12 \\
\hline $\begin{array}{l}\text { 3x4 CCM computed } \\
\text { using synthetic color } \\
\text { chart }\end{array}$ & 27.60 & 42.5 & 5.2 & 16.4 & 0.27 & 0.01 \\
\hline
\end{tabular}

Visual and qualitative comparison is shown on Figure 10. On the upper right corner sRGB image computed using standard observer functions is displayed for reference. The first row shows the case of photographic sensor (RGB channels with infrared filter), on left the raw image, on right after white balance and color correction using CCM evaluation on MCC. The second row presents images for RGBW sensor under consideration (no IR filter), from left to right: raw image, image corrected by CCM computed for RGB equivalent photographic sensors (same as in first row), image corrected by $3 \times 4$ CCM computed only on MCC patches and image corrected by 3x4 CCM computed on our SCC. Qualitatively, this last image shows acceptable hue and saturation on the scene.

\footnotetext{
$\dagger$ With $\Delta E_{2000}^{\max }=6.75, \Delta E_{2000}^{\text {mean }}=2.19, \Delta E_{2000}^{\min }=0.48$.
} 


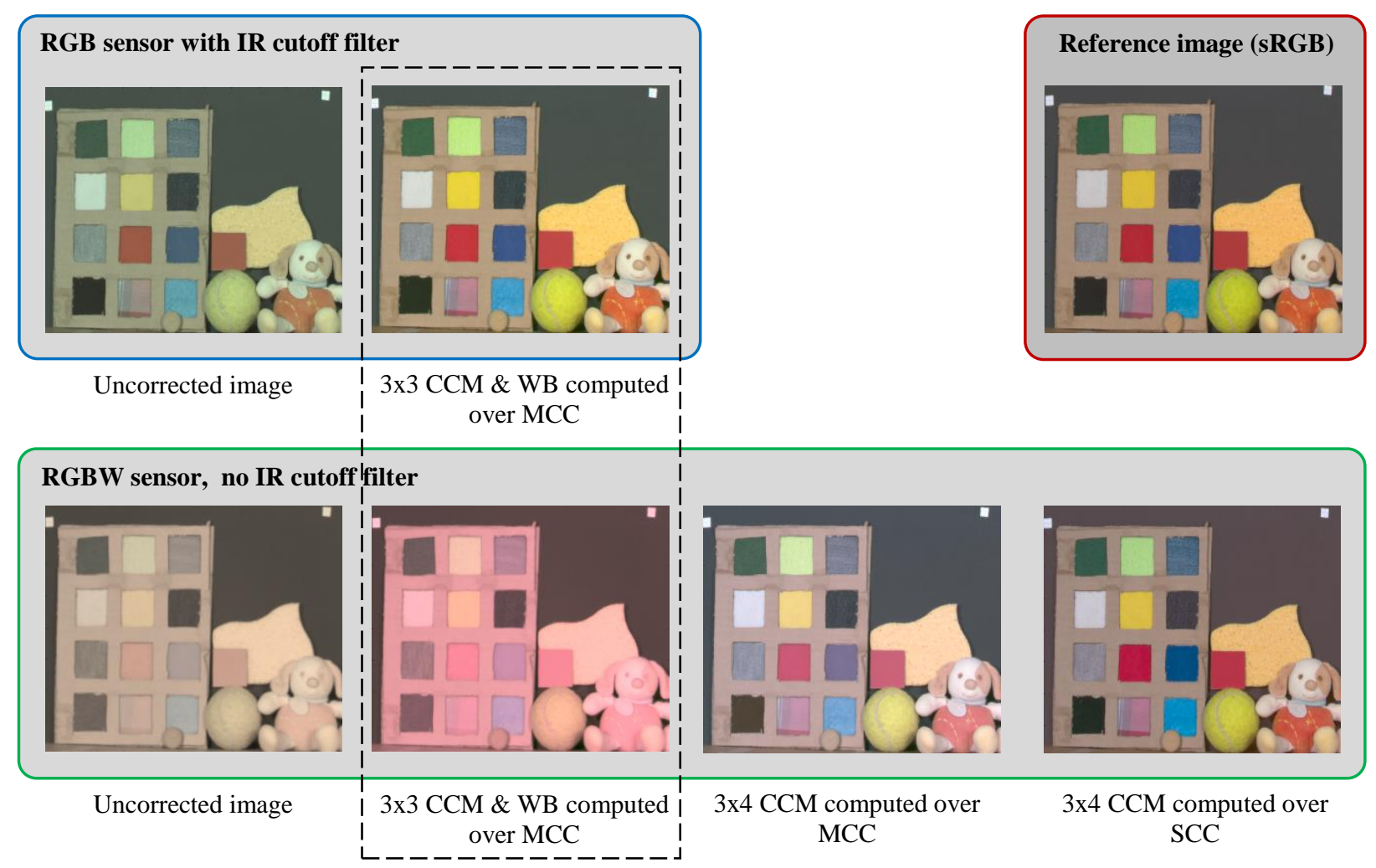

Figure 10: Comparison of color correction performance between classical visible case (RGB camera with IR cutoff filter) and low light sensor (RGBW channels without IR cutoff filter)

\section{CONCLUSION}

We proposed a new method to properly restitute colors using a sensor designed for low light level. For general application, the color correction matrix is computed with a linear least square minimization over the 24 patches of the MacBeth ColorChecker. For low light level video application, the infrared cutoff filter is removed from the sensor and a new white channel is added to increase the received signal (and signal to noise ratio). Macbeth Color Checker is no longer adapted to such VIS-NIR acquisition. We designed a Synthetic Color Chart based on 200,000 reflectance spectra collected from open access or directly acquired VIS-NIR hyperspectral images. A new $\overline{l r}$ function inspired from $\bar{y}$ CIE standard observer function is used to manage the infrared part of these spectra. In the same way, a new $c_{i r}$ function is derived to project spectra into a new $L * a * b * c_{i r}$ space inspired from CIE $L^{*} a * b *$ space. In this 4D space we select a limited number of points which are uniformly distributed. These 1498 reflectance spectra constitute our new Synthetic Color Chart, allowing to compute color correction matrix taking into account not only the visible part of the spectrum but also the near-infrared part. Finally, a performance analysis is carried out on the Teledyne-E2V Onyx EV76C664AMT-RTR. We verify that SNR is increased using the low light configuration of the sensor, then we compare color restitution performance between a $3 \times 4$ CCM computed over the classical MCC or over our synthetic color chart. The results are illustrated and a CIE $\Delta \mathrm{E}_{2000}$ computation over the synthetic chart showing that color restitution under low light conditions is better when CCM is computed using it than using MCC.

This is a prior study and we plan to investigate other definition for $\bar{r}$ and $c_{i r}$, but also the $L^{*} a^{*} b^{*} c_{i r}$ space sampling and CCM calculation. Another perspective is to design a physical color chart, perhaps containing less patches, instead of this synthetic color chart in order to practice real tests with commercial VIS-NIR color sensors.

\section{ACKNOWLEGMENT}

We would like to acknowledge B.Diaspara, P.Feyrère, P.Kuntz and T.Ligozat from Teledyne-E2V for the fruitful discussions on the topic. 


\section{REFERENCES}

[1] M. Rafinazari, E. Dubois, "Demosaicking algorithms for RGBW color filter arrays", Proc SPIE, vol. $9395,2016$.

[2] S. Bianco, et al., "Color correction pipeline optimization for digital cameras", J. Electron. Imaging, vol. 22, 2, 2013.

[3] H. Teranaka, et al., "Single-Sensor RGB and NIR Image Acquisition: Toward Optimal Performance by Taking Account of CFA Pattern, Demosaicking, and Color Correction”, Electronic Imaging, vol. 2016, 18, 2016.

[4] "Color Management Solutions and Products |X-Rite", https://www.xrite.com/.

[5] J. Farrell, Image Systems Simulation, ISBN 978-0-470-51059-9, 2015.

[6] T. Skauli, J. Farrell, "A collection of hyperspectral images for imaging systems research", Proc SPIE, vol. 8660 , 2013.

[7] M. Parmar, et al., "A database of high dynamic range visible and near-infrared multispectral images", Proc SPIE, vol. 6817,2008

[8] “Scene Database 3: 380 - 1080 nm | Imageval”, http://www.imageval.com/scene-database-3-380-1080-nm/.

[9] "USGS.gov | Science for a changing world", https://www.usgs.gov/.

[10] S. K. Shevell, The Science of Color, Second edition. ISBN 9780444512512, 2003.

[11] Y. Ohno, "CIE Fundamentals for Color Measurements", in NIST, vol. 16, 2000.

[12] A. Kim, et al., "Recovering the colors of objects from multiple near-IR images", J. Opt. Soc. Korea, vol. 19, 1, 2015.

[13] C. Mornet, et al., "Toward a Quantitative Visual Noise Evaluation of Sensors and Image Processing Pipes", Proc SPIE, vol. 7876, 2011.

[14] C. Mornet, et al., "Evaluation of color error and noise on simulated images", Proc SPIE, vol. 7537, 2010. 